\title{
Research on Enterprise Data Integration Architecture Based on Cloud Platform
}

\author{
Wei-wei Zhang, Heng-liang Wu \\ School of Management Science and Engineering, Shandong Institute of Business and Technology, Yantai, China
}

\begin{abstract}
On the basis of SODIA and VDB systems, this paper presents a new data integration system called Service Oriented Data Integration Architecture based on Hadoop (SODIH), which is constructed according to SOA principles. SODIH adopts Web Service technique in communication, and runs on Hadoop cloud platform. The experimental data shows that the proposed system can significantly improve the performance of data integration system during dealing with large data sets, and has the features of dynamicity, flexibility, loosely-coupled, extensibility, etc.
\end{abstract}

Keywords—data integration, Hadoop, Web Service, SOA

\section{基于云平台的企业数据集成框架构建}

张巍巍 吴恒亮

山东工商学院管理科学与工程学院, 烟台, 山东, 中国

摘 要 本文综合运用几种比较流行的计算机技术, 在 SODIA 和 VDB 框架基础上提出了一种基于分布式处理框架的面向服务的数 据集成框架 (SODIH), 它遵循了 SOA 的设计原则和规范, 以 Web Service 作为通信技术, 基于 Hadoop 分布式处理框架, 运行在云计 算平台上。通过实验证明, 该系统在处理大数据集时显著提高了数据集成系统的执行效率, 同时具有动态性、灵活性、低耦合和易扩 展等特点。

关键词 数据集成, Hadoop, Web Service, SOA

\section{1. 引言}

随着计算机技术在我国企业中的普及和发展, 企业信 息化程度和水平越来越高, 但由于认识、观念或技术上的 问题, 企业信息化建设中缺少系统总体规划, 导致企业中 大量异构、运行在不同软硬件平台上的信息系统同时存在， 它们之间彼此孤立、相互封闭，无法实现数据交流和共享。 随着企业信息化建设的不断深入，这些系统以及与企业外 部系统之间的信息交流需求变得日益强烈, 急需对现有的 信息系统进行集成，从而打破“信息孤岛”，实现信息共享， 数据集成的概念由此产生[1]。

数据集成是指把不同来源、不同格式以及不同特点和 性质的数据从逻辑上或物理上实现有机地集中, 从而帮助 企业实现真正的数据共享。数据集成的重点和难点在于如
何解决数据的异构性、分布性和自治性等问题。目前, 在 数据集成领域, 已有很多国内外学者和研究机构提出了相 对成熟的数据集成框架, 并在企业中得到了较好的应用。 但随着 “大数据”时代的到来, 已有的数据集成框架已经无 法满足企业信息化的需求, 急需新的框架来解决数据集成 系统的大数据集处理问题。本文在已有的数据集成框架基 础之上, 结合 Web Service、SOA、Hadoop 分布式处理框架 以及云计算等多项技术，提出了一种适合大数据集处理的 面向服务的数据集成框架。

2. 数据集成的相关研究

研究数据集成的主要目的是让用户能从大量异构、分

国家自然科学基金项目（71273158); 教育部人文社会科学研究规划基金项目（13YJA630100）; 山东省高校人文社会科学研究计划 项目 (J10WG64) 
布式的数据源中查询自己所需要的信息。为此, 很多学者 采取不同的方式研究了许多数据集成方法, 比较著名的包 括全局模式集成法 (Global-as-View, 简称 GAV) 和局部模 式集成法（Local-as-View, 简称 LAV) [2]。随着面向服务 架构 (SOA) [1][3]和软件即服务（Software as a Service, 简称 SaaS）概念的提出，一些基于“服务”的数据集成架构 相继提出, 比如面向服务的数据集成架构 (Service Oriented Data Integration Architecture, 简称 SODIA）[4]。SODIA 设 计的初衷是为了帮助用户从大量独立、异构和分布式的数 据源中提取出所需要的信息, 并能够采用统一的视图呈现 给用户, 从而为用户提供统一的数据访问机制, 实现对异 构、分布式数据源的统一访问。

在 SODIA 系统中, 服务提供者首先需要将数据源作为 数据访问服务发布到服务注册中心, 当服务被调用时可以 实现动态绑定, 从而获取所需要的数据, 服务执行完毕后 再实现服务分离。采取这种机制, 可以有效降低在组织结 构、后端数据源、数据结构以及语义等发生改变时的系统 维护成本。为达到此目的, 系统必须能够对那些数据访问 服务实现语义上的准确描述和访问。然而, SODIA 系统将 源数据元素到集成数据元素的映射以及对数据访问服务的 语义描述任务都交给了服务提供者, 结果无法保证服务能 被准确地识别和调用, 因为我们无法保证每个服务提供者 都能够用准确的术语对每个数据访问服务进行描述。

另一方面, 随着数据源数量爆炸性的增长以及云计算、 网格计算技术的出现, 数据的分布式处理越来越受到学者 的关注, 陆续出现了一些专门处理分布式大数据集的系统 框架, 比如基于 Hadoop 的虚拟数据库系统 (VDB) [5], 它利用 Hadoop 框架的并行、分布式处理能力实现了在异构 大数据集上的查询处理。

综合 SODIA 和 VDB 两种系统的技术特点, 本文提出 了一种更加动态、灵活、高效的数据集成框架, 称为基于 Hadoop 云平台的面向服务的数据集成系统 (以下简称为 SODIH), 它综合了其它两种系统的优点, 并弥补了它们各 自的缺陷, 表现出更多的技术优势。

\section{3. 基于云平台的企业数据集成框架设计}

如图 1 所示, SODIH 系统是由若干处在不同交互层上、 相互协作的服务组成的, 该设计方案遵循了 SOA 的服务设 计原则, 同时建立在 Hadoop 分布式处理框架基础之上。此 外, 该系统采用了迟绑定技术, 从而使数据集成系统具有 更好的动态性和灵活性。SODIH 系统的主要包括以下几个 模块:

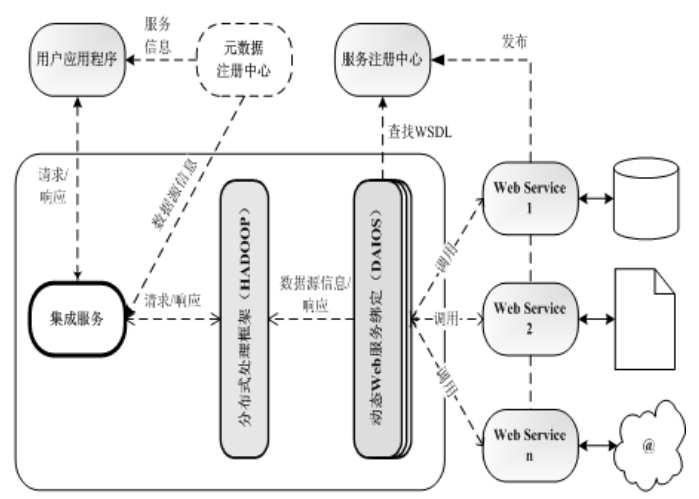

图1 基于云平台的企业数据集成框架

\section{1 动态 Web 服务绑定模块}

该模块在框架中主要负责对 Web 服务的动态绑定。系 统开发中, 我们参考使用了 DAIOS 框架[6]来完成 Web 服 务

动态绑定, 由于 DAIOS 采取消息交换机制进行通信, 而不 像目前一些框架中使用预编译的服务访问组件。因此, 它 在一定程度上增强了数据集成系统的动态性和灵活性, 而 且还能解决统一的数据类型转换问题。绑定时所使用的 Web 服务信息统一采用 Web 服务描述语言 (WSDL) 来描 述[7], 当服务请求者发出请求时, 服务端根据用户提供的 服务信息，去查找 WSDL 对应的 Web 服务并进行调用，从 而实现动态绑定。

\section{2 分布式处理框架}

分布式处理的含义是把一个工作任务分解成若干子任 务, 并交由多个工作节点同时并行处理, 任务完成后再将 每个节点上的处理结果聚集、合并起来, 最终将结果返回 给用户。在 “大数据”时代, 分布式处理将是数据处理的必 然选择, 通过它可以显著提高数据搜索以及数据处理的速 度和性能。本框架选取 Hadoop 作为分布式处理框架 [8], 它是由 Apache 基金会开发的一个开源项目, 通过它可以轻 松实现在大量服务器上对 “大数据”应用程序的分布式数据 处理。Hadoop 主要包括 HDFS 和 MapReduce 两部分。其中, MapReduce 由 Map 和 Reduce 两个动词组成, “Map (映射)” 表示将一个工作任务分解成多个子任务, “Reduce（化简）” 表示将分解后的子任务处理结果合并起来, 并得到最终结 果。

\section{3 元数据注册中心}

Web 服务元数据描述了访问某个特定服务所需要的描 述信息, 元数据注册中心负责对 Web 服务元数据进行统一 
存储和管理, 同时它还存储了系统中有关数据源的元数据 信息, 用户应用程序和集成服务模块可以将从中获取所需 要的信息。元数据注册可以根据企业情况使用数据库或采 取文件系统存储来完成。

\section{$3.4 \mathrm{Web}$ 服务注册中心}

Web 服务注册中心在该系统框架中负责 Web 服务信息 存储和查询服务。同样, Web 服务信息的存储也可以采用 数据库存储或文件系统存储等; 查找服务是服务注册中心 的一个重要功能, 它需要支持高效的查询, 服务请求者通 过WSDL 可以快速获取 Web 服务的功能以及使用说明等信 息。

\section{5 集成服务模块}

图 1 中的集成服务模块属于业务领域层服务, 它负责 数据集成系统与用户应用程序的交互。用户通过此模块可 以快速查找到系统中存储的数据源和 Web 服务信息。同时, 它还负责获取和聚集系统返回的处理结果, 并对用户作出 最终响应。

\section{4. 实验分析}

为验证本框架的有效性, 本文对 SODIA 和 SODIH 框 架的性能表现进行了对比测试。SODIH 测试在云计算环境 下进行, 基于亚马逊的弹性计算云平台 (Amazon EC2), 使用亚马逊的 Elastic MapReduce (Amazon EMR) 服务来 完成[9]。Amazon EMR 是一种 Web 服务, 它使用 Hadoop 框架作为其分布式处理引擎, 通过在 Amazon EC2 上架构 Hadoop 框架来为用户提供大数据的处理服务, 即在 EC2 实 例集群上运行 Map/Reduce 任务。

\section{1 单节点测试}

因为数据集成系统的执行效率与数据源数量有着直接 关系, 所以, 在两种测试中我们都测试并分析了系统在不 同数据源数量下的性能表现。比如, 在单节点测试中, 我 们设计了 6 种不同大小的数据源数量 (5-10000), SODIA 和 SODIH 系统的实验结果如图 2 所示。

从图中的实验数据来看, $\mathrm{SODIH}$ 在数据源数量比较小 (5-50) 时性能表现一般。但随着数据源数量的不断增大 (500-10000), 两个系统的执行效率差距也不断被拉开, 说明即使在单节点模式下, SODIH 系统在处理大数据时性 能也要优于 SODIA, 主要是因为 SODIH 采用了 Hadoop 框 架, 任务被分解成多个 Map 子任务, 采取并行处理技术, 虽然用同一个 Java 虚拟机, 执行效率也有比较明显的提升。

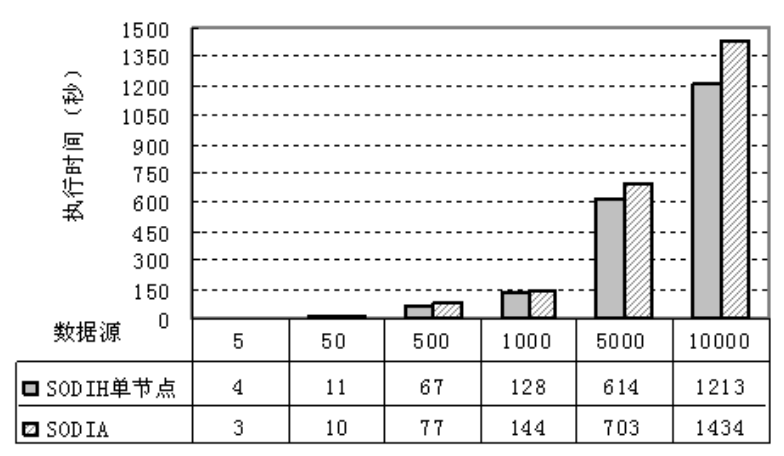

图2 SODIH和SODIA的单节点测试

\section{2 云测试}

为完成云测试, 我们将 SODIH 系统部署到 Amazon EMR 上, 并在 Amazon EC2 平台上运行 Map/Reduce 任务, 使用的 Amazon EC2 实例类型为小型实例 (m1.small)。

考虑到系统性能不仅与数据源数量有关, 还与 Hadoop 集群大小有关, 测试时我们设计了三种不同的 Hadoop 集群 配置方案:

(1) 方案 A: Hadoop 集群包括 1 个主节点和 2 个从节 点;

(2) 方案 B: Hadoop 集群包括 1 个主节点和 3 个从节 点;

(3) 方案 C: Hadoop 集群包括 1 个主节点和 4 个从节 点。

同时, 我们对 Hadoop 集群参数进行了如下调整:

(1) 在每个集群中, 输入的数据源文件被分割成了 $\mathrm{n}-1$ 个切片, 其中 $\mathrm{n}$ 代表集群中节点的数量, 即主节点不执行 计算任务, 只有从节点执行处理;

(2) 在每个集群中, 数据副本系数被设为最小值, 即 dfs.replication $=1$;

（3）在每个集群中, 为保证任务能被均匀的分配到每 个工作节点上, 每个节点的最大 Map 任务数被设置为 1 , 即 mapred.tasktracker.map.tasks.maximum $=1$ 。

具体测试结果如图 3 所示, 下面将对三种不同的测试 方案进行对比分析:

(1) 方案 A: 从图中可以看出, 数据源数量在 $5-50$ 区间时方案 $\mathrm{A}$ 具有较明显的优势, 执行时间最短, 因为在 该区间它使用的 Hadoop 集群节点数最少, 所使用的集群守 护进程数量最少, 因此耗费资源相对较少, 执行速度相对 较快。此时由于数据源数量较小, 分布式处理的优势还未 显现出来。但随着数据源数量的大幅增加, 其处理速度也 逐渐被方案 $\mathrm{B}$ 和 $\mathrm{C}$ 赶超。 


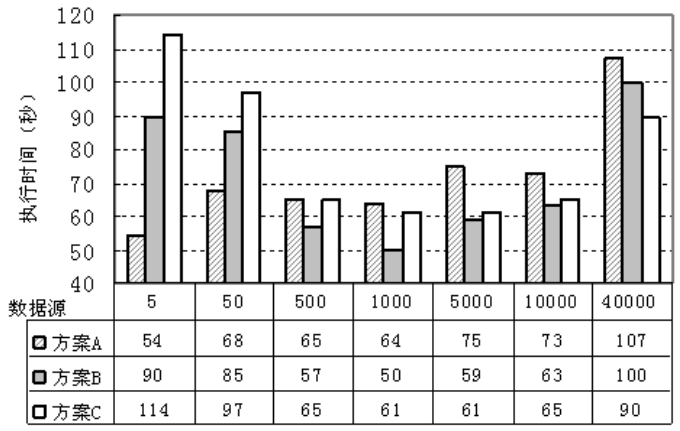

图3 基于不同Hadoop集群大小的SODIH云测试

（2）方案 B：图中显示数据源数量在 500-10000 区间 时方案 B 的执行速度最快, 说明在该区间内采用方案 B 中 的集群节点数量已经能很好地满足该系统分布式处理的需 要, 而节点耗费的资源相对较少, 执行速度相对较快; 而 在数据源数量特别大 (40000 以上) 时, 它的性能就明显不 如比其节点数量更多的方案 $\mathrm{C}$ 。

(3) 方案 $\mathrm{C}$ : 与方案 $\mathrm{B}$ 类似, 数据源数量越小, 方案 $\mathrm{C}$ 的系统性能表现越差, 而随着数据源数量的不断增加, 其性能也不断得到提升。当数据源数量大于 40000 时其执 行速度已明显超过方案 B, 说明数据源数量在进入 40000 以上的区间时, Hadoop 集群节点数需要增加到方案 $\mathrm{C}$ 中的 数量, 系统才能更好地发挥出分布式处理的优势。

\section{3 基于 Hadoop 云平台的 SODIH 系统与 SODIA 系统比较}

最后, 我们将上面云测试中的方案 $\mathrm{A}$ 与 SODIA 系统进 行了比较, 如图 4 所示。从图中可以看出, 当数据源数量 超过 500 时, 使用 Hadoop 集群的 SODIH 系统执行速度开 始超过 SODIA 系统, 而且随着数据源数量的不断增大, 两 个系统的执行效率差距变得越来越悬殊。当数据源数量增 长到 10000 时, SODIH 系统的执行速度已将近是 SODIA 系 统的 20 倍, 这充分体现出基于分布式处理的数据集成系统 在处理大数据集时的速度优势。

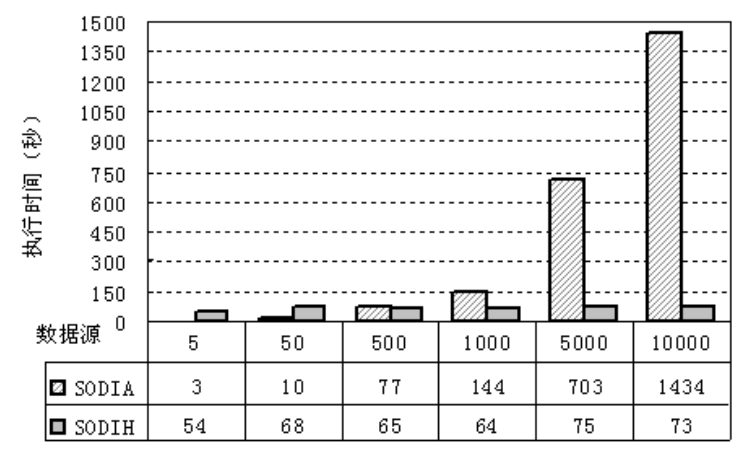

图4 SODIH（方案A）与SODIA系统的比较

\section{5. 结语}

当前, “信息孤岛”的存在已严重影响了我国企业信息 化发展的进程, 企业急需通过数据集成来打破孤岛, 将企 业内及外部系统联通起来, 实现信息共享。但由于企业现 存信息系统具有异构性、自治性和分布性等特点, 再加上 “大数据”问题的出现, 使得建立快速高效的数据集成系统 愈加困难。本文在已有数据集成系统的基础上, 提出了一 个基于 Web 服务、SOA 和 Hadoop 云平台的数据集成框架, 它综合了几种技术的优点, 具有低耦合性、易扩展、可移 植性、高可靠性以及动态性等特点。经实验测试表明, 本 框架在处理大数据集时有着比较明显的技术优势, 可以大 大提升企业数据集成系统处理大数据的能力。

\section{参考文献(Referencces)}

[1] Haibo Mao. Study of Digital Resource Integration Based on SOA. Journal of Intelligence, 2010,(06): 177-179.

[2] Guixiang Zhang. Research on the Mode Integration of the Integration System of Heterogeneous Data Sources. Microcomputer Information, 2007, (15): 233-235.

[3] Yucheng Wu, Jinquan Zhu. Research on Data Integration Based on SOA and Web Services in the Digital Library of University. Information Science, 2012, (07): 1030-1033.

[4] F. Zhu, M. Turner, I. Kotsiopoulos, K. Bennett, M. Russell, D.Budgena, P. Breretona, J. Keane, P. Layzell, M. Rigby, J. Xu, Dynamic data integration using web services, in: IEEE International Conference on Web Services, 2004: 262-269.

[5] S. Sathya, M. Jose, Application of hadoop mapreduce technique to virtual database system design, in: 2011 International Conference on Emerging Trends in Electrical and Computer Technology (ICETECT), 2011: 892-896.

[6] P. Leitner, F. Rosenberg, S. Dustdar, Daios: efficient dynamic web service invocation. IEEE Internet Computing, 2009, 13(3): $72-80$.

[7] Shi Lin. WSDL Document Structure Analysis in Web Service. Software, 2012, (10): 142-143.

[8] Linshan Ma, Qingfeng Zhao, Xinguo Xiao. Research on Mobile Cloud Information Services Model Based on Hadoop Platform. Information Science, 2013, (04): 28-32.

[9] Jinlong Li, Jie Zhu, Dianpeng Liang. A Solution of HPC Platform based Amazon EC2. Computer Knowledge and Technology, 2011,(28): 7024-7026. 